\title{
The Role of Real Time Ultrasound in Differentiating between Osteomyelitis and Tumour in Long Bones
}

\author{
J George, FRCR, SC Teo, MBBS, M Adan, MRAD (UM) \\ Department of Biomedical Imaging, University Malaya Medical Centre, Kuala Lumpur, Malaysia
}

\begin{abstract}
Aim: This study was designed to evaluate the use of ultrasound in diagnosis of infection and tumour of long bones.

Methodology: Patients referred from the orthopaedic unit with doubt regarding long bone clinical and/or radiological signs which could be tumour or infection were enrolled in this study. Analysis of ultrasound characteristics included presence of pericortical fluid over normal cortex well away from the primary lesion, wavy contour sign (fluid tracking in and out muscle planes), subperiosteal fluid and soft tissue mass displacing adjacent muscle planes.
\end{abstract}

Results: Fourteen out of 15 patients with confirmed osteomyelitis were diagnosed by ultrasound examination. Ultrasound as a diagnostic tool has a sensitivity of 93\% and specificity of $100 \%$. The most accurate indicator was pericortical fluid noted up to several centimetres from the long bone abnormality seen on plain x-rays.

Conclusion: Ultrasound is a safe, fast, cost-effective imaging modality that can play an important role in diagnosis of osteomyelitis as it then serves as a tool for ultrasound guided aspiration.

\section{Key Words:}

Osteomyelitis, Sonographic findings, Pericortical fluid, Wavy contour sign, Subperiosteal fluid

\section{INTRODUCTION}

Clinical features of osteomyelitis may be non-specific rendering accurate diagnosis on clinical and plain radiography not possible. Delay in treatment of osteomyelitis may result in complications and morbidity, so early diagnosis is essential to improve outcomes for this infection. Ultrasound has been used to investigate clinically suspected osteomyelitis ${ }^{1}$. Skeletal malignancies such as Ewing's sarcoma, osteosarcoma, myeloma or metastatic neuroblastoma can mimic osteomyelitis on plain radiographs ${ }^{2,3}$. Alternative imaging modalities including computerized tomography and magnetic resonance imaging have been recommended for this purpose ${ }^{2}$.

Ultrasound is able to detect early stage osteomyelitis and perhaps allow for non-operative treatment in selected advanced cases ${ }^{1,4}$. However, the specific role of ultrasound in the sequential imaging of osteomyelitis for skeletal infection has yet to be established. This prospective study was therefore undertaken to define the role of ultrasound in the differential diagnosis of osteomyelitis, soft tissue infection, and skeletal tumours.

\section{MATERIALS AND METHODS}

\section{Study Sample}

Thirty-one consecutive patients referred from the orthopaedic surgery unit of our institution for suspected long bone infection were included in this prospective study. The study took place over two years from 2002-2004. Demographic data and clinical information were collected. Standard anteroposterior and lateral radiographs taken at the time of admission were reviewed by the researchers. Radiographic features observed included bony changes such periosteal reaction, new bone formation, localized osteoporosis and presence of lytic areas. Investigators also took note of soft tissue changes including swelling near the metaphysis, displacement of muscle planes and obliteration of lucency between muscle planes. The ethics committee of the institution approved this study.

\section{Ultrasonography}

Ultrasound examination (using an Advanced Technology Laboratories (ATL) 3000 machine with a high frequency (7.5MHz0 linear array) transducer) was performed as soon after presentation as possible, preferably on the day of presentation to the hospital. Scanning included both transverse and sagittal sections and the entire circumference of the extremity was scanned. Patients were positioned in supine and/or prone position for optimal visualization of the involved region Ultrasound of the contralateral limb was used for comparison. 
All scan findings were recorded utilizing the following appearance on ultrasound.

(i) Hypoechoic periosseous fluid collection( pericortical fluid over normal cortex well away from the primary area of involvement.

(ii) Fluid tracking in and out of muscle planes from the cloaca (wavy contour sign).

(iii) Hypoechoic collection away from bone, entirely within the muscular plane - considered as soft tissue abscess

(iv) Increased subcutaneous tissue thickenss without any fulid - considered as cellulitis.

(v) Periosseous solid mass, different from muscle echogenicity - considered as not osteomyelitis but tumour displacing soft tissues

\section{Magnetic Resonance Imaging (MRI)}

MRI was performed in selected patients. T1-weighted, T2weighted and STIR sequences were conducted and changes recorded, either in sagittal, coronal or axial views depending on the site of disease involvement. Osteomyelitis is revealed at low signal intensity on $\mathrm{T} 1 \mathrm{~W}$, and high signal intensity on T2W and STIR sequences ${ }^{6}$. MRI can also confirm subperiosteal fluid collection and the extent of the disease. Comparison and correlation with the ultrasound findings were made.

\section{Bone scan}

For some patients had bone scan was performed as an alternative to MRI (i.e., if patients was not able to tolerate MRI procedure due to claustrophobia).

\section{Statistical analysis}

Statistical analysis was performed using SPSS. Sensitivity, specificity and accuracy for each of the radiological signs was calculated with the actual confirmation of diagnosis of osteomyelitis by MRI, open debridement or successful therapy with antibiotics.

\section{RESULTS}

Of the 31 study participants, $22(71 \%)$ were male and 9 $(29 \%)$ were female. Ethnicity of the study is as follows: 12 Malays (39\%), 10 Chinese (32\%) and 9 Indians (29\%). The age of study subjects ranged from 3 to $64 \mathrm{y}$, with a mean of $27 \mathrm{y}$. More than half of the patients (55\%) were children or young adults between 1 to $20 \mathrm{y}$ (Table I). Osteomyelitis was confirmed in 15 cases based on clinical findings, supplemented with MRI in 6 patients, biopsy and surgical drainage in 8 patients and bone scan in one patient. All osteomyelitis patients responded to antibiotic treatment.

From the 31 sets of early plain radiographs, 9 (29\%) were reported as normal and $22(71 \%)$ were considered abnormal. On the final evaluation of these 9 patients, 2 had osteomyelitis, 5 had cellulitis and 2 had soft tissue abscesses. For the remaining 22 patients with abnormal plain radiographs, 13 were later confirmed to have osteomyelitis.

Ultrasound examinations (Table II) of the 31 patients revealed diagnoses of $14(45 \%)$ cases of osteomyelitis, 6 (19\%) with cellultis , $2(6 \%)$ soft tissue abscesses and 9 (29\%) periosseous solid masses (not osteomyelitis). For the 6 patients reported to have cellulitis, 3 were further examined using MRI. MRI confirmed that one of the three was found to have osteomyelitis, and so had demonstrated a false negative for ultrasound. Due to technical difficulties, MRI was not available for the remaining 3 patients. All osteomyelitis patients were treated with antibiotics and recovered without complication. The 2 patients with soft tissue abscesses diagnosed by ultrasound had MRI confirmation of the diagnosis. For the other 9 patients with periosseous solid masses, biopsy confirmation confirmed that these masses were not osteomyelitis.

During ultrasound examination, we also observed other sonographic features described earlier and analyzed them in relation to the final diagnosis. Figures 1 and 2 show two such examples, Figure 1 shows subperiosteal fluid and Figure 2 fluid extending over normal cortex well away from the original site of the lesion. Table III summarizes the statistical analysis of these sonographic features in relation to the osteomyelitis.

\section{Statistical analysis}

Ultrasound diagnosed osteomyelitis of long bones with $93 \%$ sensitivity and $100 \%$ specificity. Positive predictive value (PPV) reaches $100 \%$ with a negative predictive value (NPV) of $94 \%$. Accuracy of diagnosis of osteomyelitis by ultrasound is $97 \%$.

\section{DISCUSSION}

Although early diagnosis of osteomyelitis is crucial, its clinical features may be confusing; thus a simple, safe and easily available diagnostic tool would be valuable adjunct. CT scans and MRI are sensitive in detecting early osteomyelitis, but they are relatively expensive and not as readily available in some areas of the world ${ }^{20}$. The current study demonstrates that ultrasound is able to diagnose osteomyelitis with $97 \%$ accuracy. The 2 cases of osteomyelitis that went undetected via normal radiography were indeed detected by ultrasound.

In addition to early detection of osteomyelitis, this study indicates that ultrasound is valuable in differentiating an infective from a non-infective lesion. The 9 cases in which ultrasound excluded osteomyelitis were eventually confirmed to be free from infective pathology. 
Table I: Age distribution of study participants and for those with the confirmed diagnosis of osteomyelitis.

(Abbreviation: OM, osteomyelitis)

\begin{tabular}{|c|c|c|}
\hline Age in Years & Number of Study Participants & Number of Patients with Confirmed OM \\
\hline$<1 \mathrm{y}$ & 0 & 0 \\
$1-12 \mathrm{y}$ & 12 & 7 \\
$13-20 \mathrm{y}$ & 5 & 3 \\
$<21 \mathrm{y}$ & 14 & 5 \\
Total & 31 & 15 \\
\hline
\end{tabular}

Table II: Comparison of osteomyelitis detected by ultrasound to confirmed diagnosis ( $93 \%$ sensitivity and $100 \%$ specificity for ultrasound. (Abbreviation: OM, osteomyelitis)

\begin{tabular}{|l|c|c|c|}
\hline Osteomyelitis Detection & $\begin{array}{c}\text { Confirmed } \\
\text { Positive OM }\end{array}$ & $\begin{array}{c}\text { Confirmed } \\
\text { Negative OM }\end{array}$ & TOTAL \\
\hline Positive osteomyelitis on ultrasound & 14 & 0 & 14 \\
Negative osteomyelitis on ultrasound & 1 & 16 & 17 \\
Total & 15 & 16 & 31 \\
\hline
\end{tabular}

Table III: Statistical analysis of the sonographic features in relation with osteomyelitis. (Abbreviations: PPV, positive predictive value; NPV, negative predictive value; ACC, accuracy)

\begin{tabular}{|c|c|c|c|c|c|}
\hline Sonographic Features & $\begin{array}{c}\text { Sensitivity } \\
(\%)\end{array}$ & $\begin{array}{l}\text { Specificity } \\
(\%)\end{array}$ & $\begin{array}{l}\text { PPV } \\
(\%)\end{array}$ & $\begin{array}{l}\text { NPV } \\
(\%)\end{array}$ & $\begin{array}{l}\text { ACC } \\
(\%)\end{array}$ \\
\hline $\begin{array}{l}\text { Periosseous fuid collection adjacent to normal cortex with } \\
\text { osteomyelitis }\end{array}$ & 93 & 100 & 100 & 94 & 97 \\
\hline Subperiosteal fluid with osteomyelitis & 73 & 100 & 100 & 80 & 87 \\
\hline Periosseous wavy fluid collection with osteomyelitis & 87 & 100 & 100 & 89 & 94 \\
\hline Cortical break with fluid in and out with of cloaca. & 40 & 100 & 100 & 64 & 71 \\
\hline Periosseous solid mass, not osteomyelitis(in our study) & 100 & 100 & 100 & 100 & 100 \\
\hline
\end{tabular}

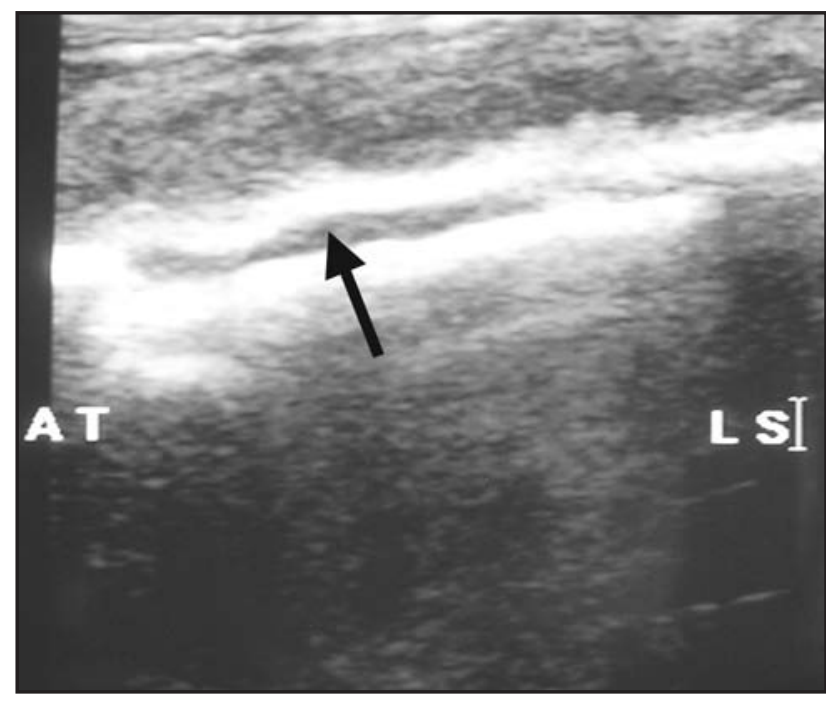

Fig. 1: Arrow points to the presence of subperiosteal fluid on ultrasound as evidenced by a hypoechoic line under the periosteum on real time ultrasound.

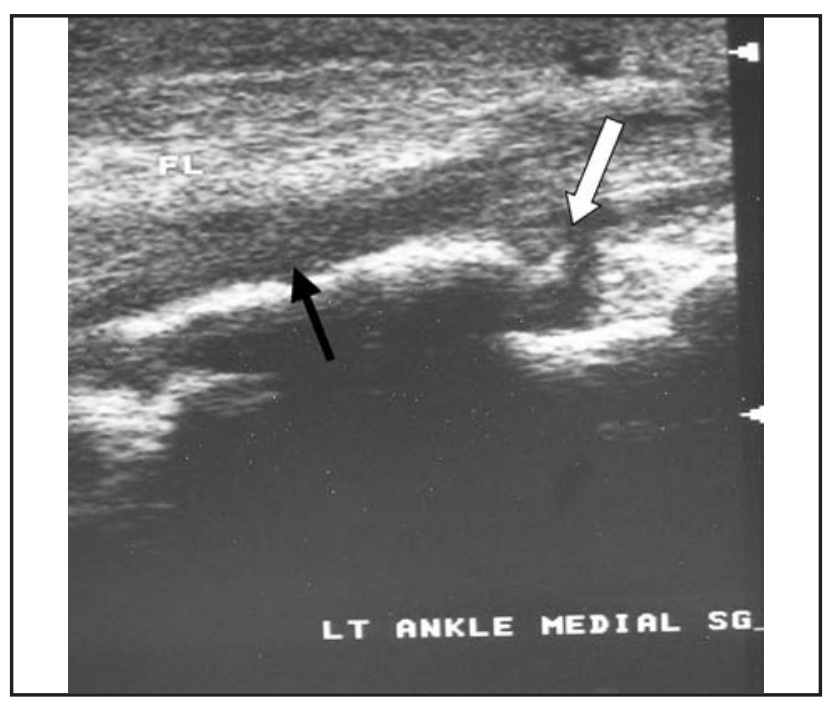

Fig. 2: Arrow shows fluid over normal cortex that stretches over several $\mathrm{cm}$ along the bone away from the cloaca (white arrow) in the long bone. This is shown by the presence of hypoechoic line over the cortex streching over the normal hyperechoic cortex which appears as a hyperechoic band. 
Ultrasound is an also excellent investigative tool to differentiate between cystic and solid masses ${ }^{21,22}$. This differentiation is very important to the clinician, as immediate management is different in both conditions. In rural areas where CT scan, MRI or nuclear medicine imaging is not available, ultrasound is an ideal alternative imaging device after plain radiography because it provides economic and noninvasive imaging of the tissue. Early diagnosis of osteomyelitis enables the clinician to initiate treatment before extensive bone and tissue injury occur. Additionally, ultrasound can also be used to guide the placement of the needle tip during diagnostic or therapeutic aspiration for osteomyelitis.

The presence of periosseous (pericortical) fluid collection over normal bone away from the primary lesion appears to be the best sonographic indicator for diagnosis osteomyelitis by ultrasound. A demonstration of fluid collection contiguous to bone surface is essential for accurate sonographic diagnosis of acute osteomyelitis ${ }^{1}$. Our results revealed 93\% sensitivity, $100 \%$ specificity and $97 \%$ accuracy for this sonographic feature in diagnosing osteomyelitis. In one patient where the diagnosis of osteomyelitis was missed by ultrasound, the patient had been treated with a complete course of antibiotic prior to presentation and examination.
The medical therapy may have altered the tissue condition. Subperiosteal fluid collection is a specific (100\%) sonographic feature for osteomyelitis but not sensitive enough $(73 \%)$ for definitive diagnosis as not all the osteomyelitis cases had this feature. Subperiosteal abscess is more commonly found in children as loose attachment of the periosteum to the cortex in children enables infection to extend into the subperiosteal region ${ }^{10}$. Although periosseous wavy fluid collection and cortical break with surrounding fluid are not sufficiently sensitive indicators to diagnose osteomyelitis, these features were observed in some cases of confirmed osteomyelitis in this study.

\section{CONCLUSION}

Ultrasound is a valuable imaging tool in diagnosis of osteomyelitis in that it carries high sensitivity and specificity for accurate diagnosis. Additionally, ultrasonography provides further information on condition of the surrounding soft tissue, differentiating osteomyelitis from soft tissue infections and other non-infective bone lesions. Ultrasound has the advantage of being simple, inexpensive and freely available in most hospitals and can be performed without undue delay. 


\section{REFERENCES}

1. Desai RV, Khandelwal N, Gupta S, Mani NBS, Gill SS, Suri S, et al. Role of ultrasound in acute osteomyelitis. Asian Ocean J. Radiol. 2001; 6(3): 159-65.

2. Oudjane K, Azouz EM. Imaging of osteomyelitis in children. Radiol Clin North Am. 2001; 39(2): 251-66.

3. Nath AK, Sethu AU. Use of ultrasound in osteomyelitis. Br J Radiol. 1992; 65(776): 649-52.

4. Wright NB, Abbott GT, Carty HMC. Ultrasound in children with osteomyelitis. Clin Radiol. 1995; 50: 623-7.

5. Carek PJ, Dickerson LM, Sack JI. Diagnosis and management of osteomyelitis. Am Fam Physician. 2001; 63(12): $2413-20$.

6. Tehranzadeh J, Wong E, Wang F, Sadighpour M. Imaging of osteomyelitis in the mature skeleton. Radiol Clin North Am. 2001; 39(2): 223-50.

7. Kothari NA, Pelchoutz DJ, Meyer JS. Imaging of musculoskeletal infection. Radiol Clin North Am. 2001; 39(4): 653-71.

8. Capitanio MA, Kirpatrick JA. Early roentgen observations in acute osteomyelitis. Am J Roentgenol. 1970; 108: 488-96.

9. Kaiser S, Rosenberg M. Early detection of subperiosteal abscesses by ultrasonography. Pediatr Radiol. 1994; $24: 336-9$.

10. Cardinal E, Bureau NJ, Aubin B, Chhem RK. Role of ultrasound in musculoskeletal infection. Radiol Clin North Am. 2001; 39(2): 191-201.

11. Schlesinger AE, Hernandez RJ. Diseases of musculoskeletal system in children: Imaging with CT, sonography and MR. Am $J$ Radiol. 1992; 158: 729-41.

12. Unger E, Moldofsky P, Gatenby R. Diagnosis of osteomyelitis by MR imaging. Am J Roentgenol. 1988; 150: 605-10.

13. Reibel TW, Nasir R, Nazarenko O. The value of sonography in the detection of osteomyelitis. Pediatr Radiol. 1996; $26: 291-7$.

14. Steiner GM. The value of ultrasound in the assessment of bone. Br J Radiol. 1992; 65: 589-93.

15. Kaplan PA, Matamoros A Jr, Anderson JC. Sonography of the musculoskeletal system. Am J Radiol. 1990; 155: 237-45.

16. Chhem RK, Kaplan PA, Dussault RG. Ultrasonography of the musculoskeletal system. Radiol Clin North Am. 1994; 32 (2): 275 89.

17. Chhem RK, Schmutz GR, Huynh HH, Bui BT. Ultrasonography of a bone metastasis. Can Assoc Radiol J. 1992; 43(2): $138-40$.

18. Cleveland TJ, Peck RJ. Case Report: Chronic osteomyelitis demonstrated by high resolution ultrasonography. Clin Radiol. 1994; 49: 429-31.

19. Craig JG. Infection: Ultrasound-guided procedures. Radiol Clin North Am. 1999; 37(4): 669-78.

20. Primack SJ. Musculoskeletal ultrasound: The clinician's perspective. Radiol Clin North Am. 1999; 37(4): 617-22.

21. Mittelstaedt CA . General ultrasound- musculoskeletal and soft tissue. New York. Churchill Livingstone. 1992; 1-53.

22. Abiri MM, Kirpekar JA, Ablow RC. Osteomyelitis: detection by ultrasound. Radiology. 1989; 172: 509-11. 